\title{
THE POWER OF STRONG FOURIER SAMPLING: QUANTUM ALGORITHMS FOR AFFINE GROUPS AND HIDDEN SHIFTS*
}

\author{
CRISTOPHER MOORE ${ }^{\dagger}$, DANIEL ROCKMORE ALEXANDER RUSSELL $^{\S}$, AND \\ LEONARD J. SCHULMAN $₫$
}

\begin{abstract}
Many quantum algorithms, including Shor's celebrated factoring and discrete log algorithms, proceed by reduction to a hidden subgroup problem, in which an unknown subgroup $H$ of a group $G$ must be determined from a quantum state $\psi$ over $G$ that is uniformly supported on a left coset of $H$. These hidden subgroup problems are typically solved by Fourier sampling: the quantum Fourier transform of $\psi$ is computed and measured. When the underlying group is nonabelian, two important variants of the Fourier sampling paradigm have been identified: the weak standard method, where only representation names are measured, and the strong standard method, where full measurement (i.e., the row and column of the representation, in a suitably chosen basis, as well as its name) occurs. It has remained open whether the strong standard method is indeed stronger, that is, whether there are hidden subgroups that can be reconstructed via the strong method but not by the weak, or any other known, method. In this article, we settle this question in the affirmative. We show that hidden subgroups $H$ of the $q$-hedral groups, i.e., semidirect products $\mathbb{Z}_{q} \ltimes \mathbb{Z}_{p}$, where $q \mid(p-1)$, and in particular the affine groups $A_{p}$, can be information-theoretically reconstructed using the strong standard method. Moreover, if $|H|=p / \operatorname{polylog}(p)$, these subgroups can be fully reconstructed with a polynomial amount of quantum and classical computation. We compare our algorithms to two weaker methods that have been discussed in the literature - the "forgetful" abelian method, and measurement in a random basis-and show that both of these are weaker than the strong standard method. Thus, at least for some families of groups, it is crucial to use the full power of representation theory and nonabelian Fourier analysis, namely, to measure the highdimensional representations in an adapted basis that respects the group's subgroup structure. We apply our algorithm for the hidden subgroup problem to new families of cryptographically motivated hidden shift problems, generalizing the work of van Dam, Hallgren, and Ip on shifts of multiplicative characters. Finally, we close by proving a simple closure property for the class of groups over which the hidden subgroup problem can be solved efficiently.
\end{abstract}

Key words. quantum computation, hidden subgroup problem, Fourier analysis, group representations

AMS subject classifications. 81P68, 20C35

DOI. $10.1137 /$ S0097539705447177

1. The hidden subgroup problem. One of the principal quantum algorithmic paradigms is the use of the abelian Fourier transform to discover a function's hidden periodicities. In the examples relevant to quantum computing, an oracle function $f$ defined on an abelian group $G$ has "hidden periodicity" if there is a "hidden" subgroup $H$ of $G$ so that $f$ is precisely invariant under translation by $H$ or, equivalently, $f$ is constant on the cosets of $H$ and takes distinct values on distinct cosets. The hidden

* Received by the editors March 9, 2005; accepted for publication (in revised form) December 20, 2006; published electronically August 24, 2007. Support for this work was provided by the California Institute of Technology's Institute for Quantum Information (IQI), the Mathematical Sciences Research Institute (MSRI), the Institute for Advanced Study (IAS), NSF grants ITR-0220070, ITR-0220264, CCR-0093065, CCR-0049092, EIA-0218443, QuBIC-0218563, and CCF-0524613, the Charles Lee Powell Foundation, and the Bell Fund.

http://www.siam.org/journals/sicomp/37-3/44717.html

${ }^{\dagger}$ University of New Mexico, Albuquerque, NM 87131, and Santa Fe Institute, Santa Fe, NM 87501 (moore@cs.unm.edu)

¥Dartmouth College, Hanover, NH 03755 (rockmore@cs.dartmouth.edu).

$\S$ University of Connecticut, Storrs, CT 06269 (acr@cse.uconn.edu).

๑California Institute of Technology, Pasadena, CA 91125 (schulman@caltech.edu). 
subgroup problem is the problem of determining the subgroup $H$ from such a function. Algorithms for these problems typically adopt the approach detailed below, called Fourier sampling [3].

Step 1. Prepare two registers, the first in a uniform superposition over the elements of a group $G$ and the second with the value zero, yielding the state

$$
\psi_{1}=\frac{1}{\sqrt{|G|}} \sum_{g \in G}|g\rangle \otimes|0\rangle .
$$

Step 2. Calculate (or, if it is an oracle, query) the function $f$ defined on $G$ and XOR it with the second register. This entangles the two registers and results in the state

$$
\psi_{2}=\frac{1}{\sqrt{|G|}} \sum_{g \in G}|g\rangle \otimes|f(g)\rangle .
$$

Step 3. Measure the second register. This produces a uniform superposition over one of $f$ 's level sets, i.e., the set of group elements $g$ for which $f(g)$ takes the measured value $f_{0}$. As the level sets of $f$ are the cosets of $H$, this puts the first register in a uniform distribution over superpositions on one of those cosets, namely $\mathrm{cH}$, where $f(c)=f_{0}$ for some $f_{0}$. Moreover, it disentangles the two registers, resulting in the state $\psi_{3} \otimes\left|f_{0}\right\rangle$, where $\psi_{3}$ is a so-called coset state,

$$
\psi_{3}=|c H\rangle=\frac{1}{\sqrt{|H|}} \sum_{h \in H}|c h\rangle
$$

Alternately, since the value $f_{0}$ we observe has no bearing on the algorithm, we can use the formulation in which the environment, rather than the user, measures $f$. In that case, tracing over $f$ yields a mixed state with density matrix

$$
\rho_{H}=\frac{1}{[G: H]} \sum_{f_{0}}\left|\psi_{3}\right\rangle\left\langle\psi_{3}\left|=\frac{1}{|G|} \sum_{c}\right| c H\right\rangle\langle c H|,
$$

i.e., a classical mixture consisting of one pure state $\psi_{3}$ for each coset. Kuperberg refers to this as the coherent hidden subgroup problem [18].

Step 4 . Carry out the quantum Fourier transform on $\psi_{3}$ or $\rho_{H}$ and measure the result.

For example, in Simon's algorithm [26], the "ambient" group $G$ over which the Fourier transform is performed is $\mathbb{Z}_{2}^{n}, f$ is an oracle with the promise that $f(x)=f(x+$ $y$ ) for some $y$, and $H=\{0, y\}$ is a subgroup of order 2. In Shor's factoring algorithm [25] $G$ is the group $\mathbb{Z}_{n}^{*}$, where $n$ is the number we wish to factor, $f(x)=r^{x} \bmod n$ for a random $r<n$, and $H$ is the subgroup of $\mathbb{Z}_{n}^{*}$ of index order $(r)$. (However, since $\left|\mathbb{Z}_{n}^{*}\right|$ is unknown, Shor's algorithm actually performs the transform over $\mathbb{Z}_{q}$, where $q$ is polynomially bounded by $n$; see $[25]$ or $[11,12]$.)

These are all abelian instances of the hidden subgroup problem (HSP). Interest in nonabelian versions of the HSP evolved from the relation to the elusive Graph Automorphism problem: if one could efficiently solve the HSP over the symmetric group $S_{n}$, this would yield an efficient quantum algorithm for graph automorphism (see, e.g., Jozsa [16] for a review). This was the impetus behind the development of the first nonabelian quantum Fourier transform [2] and is, in part, the reason that the nonabelian HSP has remained such an active area of research in quantum algorithms. 
In general, we will say that the HSP for a family of groups $G$ has a Fourier sampling algorithm if a procedure similar to that outlined above works. Specifically, the algorithm prepares a coset state as defined above,

$$
|c H\rangle=\frac{1}{\sqrt{|H|}} \sum_{h \in H}|c h\rangle,
$$

over a random coset $c H$ of the hidden subgroup $H$, computes the (quantum) Fourier transform of this state, and measures the result. After a polynomial number of such trials, a polynomial amount of classical computation, and, perhaps, a polynomial number of classical queries to the function $h$ to confirm the result, the algorithm produces a set of generators for the subgroup $H$ with high probability.

When $G$ is abelian, measuring a state's Fourier transform has a clear meaning: one observes the frequency $\chi$ with probability equal to the squared magnitude of the transform at that frequency. In the case where $G$ is a nonabelian group, however, in order to define a full measurement it is necessary to select bases for each representation of $G$. (We explain this in more detail below.) The subject of this article is the relationship between this choice of basis and the information gleaned from the measurement: are some bases more useful for computation than others?

Since we are typically interested in exponentially large groups, we will take the size of our input to be $n=\log |G|$. Throughout, "polynomial" means polynomial in $n$ and thus polylogarithmic in $|G|$.

1.1. Nonabelian hidden subgroup problems. Although a number of interesting results have been obtained on the nonabelian HSP, the groups for which efficient solutions are known remain woefully few. On the positive side, Roetteler and Beth [22] give an algorithm for the wreath product $\mathbb{Z}_{2}^{k} \succ \mathbb{Z}_{2}$. Ivanyos, Magniez, and Santha [15] extend this to the more general case of semidirect products $K \ltimes \mathbb{Z}_{2}^{k}$, where $K$ is of polynomial size, and also give an algorithm for groups whose commutator subgroup is of polynomial size. Friedl, Ivanyos, Magniez, Santha, and Sen [8] solve a problem they call hidden translation and thus generalize this further to what they call "smoothly solvable" groups: these are solvable groups whose derived series is of constant length and whose abelian factors are each the direct product of an abelian group of bounded exponent and one of polynomial size. (See also section 8.)

In another vein, Ettinger and Høyer [6] show that the HSP is solvable for the dihedral groups in an information-theoretic sense; namely, a polynomial number of quantum queries to the function oracle gives enough information to reconstruct the subgroup, but the best known reconstruction algorithm takes exponential time. More generally, Ettinger, Høyer, and Knill [7] show that for arbitrary groups the HSP can be solved information-theoretically with a finite number of quantum queries. However, their algorithm calls for a quantum measurement for each possible subgroup, and since there might be $|G|^{\Omega(\log |G|)}$ of these, it requires an exponential number of quantum operations.

Our current understanding of the HSP, then, divides group families into three classes.

I. Fully reconstructible. Subgroups of a family of groups $\left\{G_{i}\right\}$ are fully reconstructible if the HSP can be solved with high probability by a quantum circuit of size polynomial in $\log \left|G_{i}\right|$.

II. Information-theoretically reconstructible. Subgroups of a family of groups $\left\{G_{i}\right\}$ are information-theoretically reconstructible if the solution to the HSP for $G_{i}$ is de- 
termined information-theoretically by the fully measured result of a quantum circuit of size polynomial in $\log \left|G_{i}\right|$.

III. Quantum information-theoretically reconstructible. Subgroups of a family of groups $\left\{G_{i}\right\}$ are quantum information-theoretically reconstructible if the solution to the HSP for $G_{i}$ is determined by the quantum state resulting from a quantum circuit of polynomial size in $\log \left|G_{i}\right|$, in the sense that there exists a positive operator-valued measurement (POVM) that yields the subgroup $H$ with constant probability but where it may or may not be possible to carry out this POVM with a quantum circuit of polynomial size.

In each case, the quantum circuit has oracle access to a function $f: G \rightarrow S$, for some set $S$, with the property that $f$ is constant on each left coset of a subgroup $H$ and distinct on distinct cosets.

In this language, then, subgroups of abelian groups are fully reconstructible, while the result of [7] shows that subgroups of arbitrary groups are quantum informationtheoretically reconstructible. The other work cited above has labored to place specific families of nonabelian groups into the more algorithmically meaningful classes I and II.

1.2. Nonabelian Fourier transforms. In this section we give a brief review of nonabelian Fourier analysis but only to the extent needed to set down notation. We refer the reader to $[9,24]$ for a more complete exposition.

Fourier analysis over a finite abelian group $A$ expresses a function $\phi: A \rightarrow \mathbb{C}$ as a linear combination of homomorphisms $\chi: A \rightarrow \mathbb{C}$. If $A=\mathbb{Z}_{p}$, for example, these are the familiar basis functions $\chi_{t}: z \mapsto \omega_{p}^{t z}$, where $\omega_{p}$ denotes the $p$ th root of unity $\mathrm{e}^{2 \pi i / p}$. Any function $\phi: A \rightarrow \mathbb{C}$ can be uniquely expressed as a linear combination of these $\chi_{t}$, and this change of basis is the Fourier transform.

When $G$ is a nonabelian group, however, this same procedure cannot work: in particular, there are not enough homomorphisms of $G$ into $\mathbb{C}$ to span the space of all $\mathbb{C}$-valued functions on $G$. To define a sufficient basis, the representation theory of finite groups considers more general functions, namely homomorphisms from $G$ into groups of unitary matrices.

A representation of a finite group $G$ is a homomorphism $\rho: G \rightarrow \mathrm{U}(d)$, where $\mathrm{U}(d)$ denotes the group of unitary $d \times d$ matrices (with entries from $\mathbb{C}$ ); the dimension $d=d_{\rho}$ is referred to as the dimension of $\rho$. If $\rho: G \rightarrow \mathrm{U}(d)$ is a representation, a subspace $W$ of $\mathbb{C}^{d}$ is said to be invariant if $\rho(g)(W) \subset W$ for all $g$. A representation is said to be irreducible if the only invariant subspaces are the trivial subspaces $\mathbb{C}^{d}$ and $\{\overrightarrow{0}\}$.

For a function $\phi: G \rightarrow \mathbb{C}$ and an irreducible representation $\rho, \hat{\phi}(\rho)$ denotes the Fourier transform of $\phi$ at $\rho$ and is defined by

$$
\hat{\phi}(\rho)=\sqrt{\frac{d_{\rho}}{|G|}} \sum_{g} \phi(g) \rho(g) .
$$

Note that $\phi$ takes values in $\mathbb{C}$ while $\rho$ is matrix-valued. It is a fact that a finite group has a finite number of distinct irreducible representations up to isomorphism (i.e., up to a unitary change of basis). The Fourier transform of a function $\phi: G \rightarrow$ $\mathbb{C}$ is then the collection of matrices $\hat{\phi}(\rho)$, taken over all nonisomorphic irreducible representations $\rho$.

Fixing a group $G$ and a subgroup $H$, we shall focus primarily on the functions 
$\varphi_{c}: G \rightarrow \mathbb{C}$ of the form

$$
\varphi_{c}(g)= \begin{cases}1 / \sqrt{|H|} & \text { if } g \in c H \\ 0 & \text { otherwise }\end{cases}
$$

corresponding to the first register of the state $\psi_{3}$ resulting from Step 3 above, which is a uniform superposition over the coset $\mathrm{cH}$. The Fourier transform of such a function is

$$
\widehat{\varphi}_{c}(\rho)=\sqrt{\frac{d_{\rho}}{|G||H|}} \rho(c) \cdot \sum_{h \in H} \rho(h) .
$$

Note, as above, that $\widehat{\varphi_{c}}(\rho)$ is a $d_{\rho} \times d_{\rho}$ matrix.

For any subgroup $H$, the sum $\sum_{h} \rho(h)$ is precisely $|H|$ times a projection operator (see, e.g., [13]); we write

$$
\sum_{h} \rho(h)=|H| \pi_{H}(\rho)
$$

With this notation, we can express $\widehat{\varphi_{c}}(\rho)$ as $\sqrt{n_{\rho}} \rho(c) \cdot \pi_{H}(\rho)$, where $n_{\rho}=d_{\rho}|H| /|G|$. For a $d \times d$ matrix $M$, we let $\|M\|$ denote the matrix norm given by

$$
\|M\|^{2}=\operatorname{tr}\left(M^{\dagger} M\right)=\sum_{i j}\left|M_{i j}\right|^{2}
$$

where $M^{\dagger}$ denotes the conjugate transpose of $M$. Then the probability that we observe the representation $\rho$ is

$$
\begin{aligned}
\left\|\widehat{\varphi_{c}}(\rho)\right\|^{2} & =\left\|\sqrt{n_{\rho}} \rho(c) \pi_{H}(\rho)\right\|^{2} \\
& =n_{\rho}\left\|\pi_{H}(\rho)\right\|^{2} \\
& =n_{\rho} \mathbf{r k} \pi_{H}(\rho),
\end{aligned}
$$

where $\mathbf{r k} \pi_{H}(\rho)$ denotes the rank of the projection operator $\pi_{H}(\rho)$. See [13] for more discussion.

1.3. Weak vs. strong sampling and the choice of basis. Hallgren, Russell, and Ta-Shma [13] show that by measuring only the names of representations - the socalled weak standard method in the terminology of [10] - it is possible to reconstruct normal subgroups (and thus solve the HSP for Hamiltonian groups, all of whose subgroups are normal). More generally, this method reconstructs the normal core of a subgroup, i.e., the intersection of all its conjugates. On the other hand, they show that this is insufficient to solve Graph Automorphism, since even in an informationtheoretic sense this method cannot distinguish between the trivial subgroup of $S_{n}$ and subgroups of order 2 consisting of the identity and an involution.

Therefore, in order to solve the HSP for nonabelian groups, we need to measure not just the name of the representation we are in but also the row and column. In order for this measurement to be well defined, we need to choose a basis for $\mathrm{U}\left(d_{\rho}\right)$ for each $\rho$. Grigni, Schulman, Vazirani, and Vazirani [10] call this the strong standard method. They show that if we measure using a uniformly random basis, then trivial and nontrivial subgroups are still information-theoretically indistinguishable. However, they leave open the question of whether the strong standard method with 
a clever choice of basis, rather than a random one, allows us to solve the HSP in nonabelian groups, yielding an algorithm for Graph Automorphism.

Indeed, from a computational perspective the representation theory of a finite group $G$ does distinguish certain "preferred" bases, those which give the matrices $\rho(g)$ unusually structured or sparse form. In particular, Moore, Rockmore, and Russell [20] showed that so-called adapted bases yield highly efficient algorithms for the quantum Fourier transform.

1.4. Contributions of this paper. As stated above, [13] and [10] leave an important open question, namely, whether there are cases where the strong standard method, with the proper choice of basis, offers an advantage over a simple abelian transform or the weak standard method. We settle this question in the affirmative. Our results deal primarily with the $q$-hedral groups, i.e., semidirect products of the form $\mathbb{Z}_{q} \ltimes \mathbb{Z}_{p}$, where $q \mid(p-1)$, and in particular the affine groups $A_{p} \cong \mathbb{Z}_{p}^{*} \ltimes \mathbb{Z}_{p}$.

We begin in section 3 by focusing on full reconstructibility. We define the hidden conjugate problem (HCP) as follows: given a group $G$, a nonnormal subgroup $H$, and a function which is promised to be constant on the cosets of some conjugate $H^{b}=b H b^{-1}$ of $H$ (and distinct on distinct cosets), determine the subgroup $H^{b}$ by finding an element $c \in G$ so that $H^{c}=H^{b}$. We adopt the above classification (fully, informationtheoretically, quantum information-theoretically) for this problem in the natural way. Then we show that given a subgroup of sufficiently small (but still exponentially large) index, hidden conjugates in $A_{p}$ are fully reconstructible (Theorem 1). This almost immediately implies that, for prime $q=(p-1) / \operatorname{polylog}(p)$, subgroups of the $q$-hedral groups $\mathbb{Z}_{q} \ltimes Z_{p}$ are fully reconstructible (Theorem 2 ).

Section 4 concerns itself with information-theoretic reconstructibility. We generalize the results of Ettinger and Høyer on the dihedral group and show that hidden conjugates of any subgroup are information-theoretically reconstructible in the affine groups and, more generally, the $q$-hedral groups for all $q$ (Theorem 3). We then show that we can identify the order, and thus the conjugacy class, of a hidden subgroup, and this implies that all subgroups of the affine and $q$-hedral groups are informationtheoretically reconstructible (Theorem 5 ).

The results of sections 3 and 4 rely crucially on measuring the high-dimensional representations of the affine and $q$-hedral groups in a well-chosen basis, namely an adapted basis that respects the group's subgroup structure. We show in section 5 that we lose information-theoretic reconstructibility if we measure in a random basis instead. Specifically, we need an exponential number of measurements to distinguish conjugates of small subgroups of $A_{p}$. This establishes for the first time that the strong standard method is indeed stronger than measuring in a random basis: some bases provide much more information about the hidden subgroup than others.

For some nonabelian groups, the HSP can be solved with a "forgetful" approach, where we erase the group's nonabelian structure and perform an abelian Fourier transform instead. In section 6 we show that this is not the case for the affine groups. Specifically, if we treat $A_{p}$ as a direct product rather than a semidirect one, its conjugate subgroups become indistinguishable.

As an application, in section 7 we consider hidden shift problems. In the setting we consider, one must reconstruct a "hidden shift" $s \in \mathbb{Z}_{p}$ from an oracle $f_{s}(x)=$ $f(x-s)$, where $f$ is any function that is constant on the (multiplicative) cosets of a known multiplicative subgroup of $\mathbb{Z}_{p}^{*}$. These functions have been studied in some depth for their pseudorandom properties, and several instances have been suggested as cryptographically strong pseudorandom generators. By associating $f_{s}$ with its 
isotropy subgroup, and using our reconstruction algorithm to find that subgroup, we give an efficient quantum algorithm for the hidden shift problem in the case where $f(x)$ is a function of $x$ 's multiplicative order $\bmod r$ for some $r=\operatorname{polylog}(p)$. This generalizes the work of van Dam, Hallgren, and Ip [4], who give an algorithm for hidden shift problems in the case where $f$ is precisely a multiplicative character.

Finally, in section 8 we show that the set of groups for which the HSP can be solved in polynomial time has the following closure property: if $\mathcal{H}=\left\{H_{n}\right\}$ is a family of groups for which we can efficiently solve the HSP and $\mathcal{K}=\left\{K_{n}\right\}$ is a family of groups for which $\left|K_{n}\right|=\operatorname{polylog}\left(\left|H_{n}\right|\right)$, we can also efficiently solve the HSP for the family $\left\{G_{n}\right\}$, where each $G_{n}$ is any extension of $K_{n}$ by $H_{n}$. This subsumes the results of [13] on Hamiltonian groups, and also those of [15] on groups with commutator subgroups of polynomial size.

We note that subsequent to this work, Bacon, Childs, and van Dam [1] found additional algorithms for the HSP in the affine groups. Their approach uses the "pretty good measurement," which they showed is optimal for certain cases of the HSP (see also [21]). Their work extends to a number of other group families, such as the Heisenberg groups.

2. The affine and $\boldsymbol{q}$-hedral groups. Let $A_{p}$ be the affine group, consisting of ordered pairs $(a, b) \in \mathbb{Z}_{p}^{*} \times \mathbb{Z}_{p}$, where $p$ is prime, under the multiplication rule $\left(a_{1}, b_{1}\right) \cdot\left(a_{2}, b_{2}\right)=\left(a_{1} a_{2}, b_{1}+a_{1} b_{2}\right)$. $A_{p}$ can be viewed as the set of affine functions $f_{(a, b)}: \mathbb{Z}_{p} \rightarrow \mathbb{Z}_{p}$ given by $f_{(a, b)}: x \mapsto a x+b$ where multiplication in $A_{p}$ is given by function composition. Structurally, $A_{p}$ is a semidirect product $\mathbb{Z}_{p}^{*} \ltimes \mathbb{Z}_{p} \cong \mathbb{Z}_{p-1} \ltimes \mathbb{Z}_{p}$. Its subgroups can be described as follows:

- Let $N \cong \mathbb{Z}_{p}$ be the normal subgroup of size $p$ consisting of elements of the form $(1, b)$. Geometrically, this is the set of affine functions with slope 1 .

- Let $H \cong \mathbb{Z}_{p}^{*} \cong \mathbb{Z}_{p-1}$ be the nonnormal subgroup of size $p-1$ consisting of the elements of the form $(a, 0)$. Geometrically, this is the set of lines passing through the origin.

- For each $b \in \mathbb{Z}_{p}$, the conjugate subgroup $H^{b}=(1, b) \cdot H \cdot(1,-b)$ consists of elements of the form $(a,(1-a) b)$. In the action on $\mathbb{Z}_{p}, H^{b}$ is the stabilizer of $b$; geometrically, $H^{b}$ is the set of lines intersecting the diagonal at $(b, b)$.

- If $a \in \mathbb{Z}_{p}^{*}$ has order $q$, where $q$ divides $p-1$, let $N_{q} \cong \mathbb{Z}_{q} \ltimes \mathbb{Z}_{p}$ be the normal subgroup consisting of all elements of the form $\left(a^{t}, b\right)$. Geometrically, $N^{q}$ is the set of lines whose slope is a power of $a$.

- Similarly, if $a \in \mathbb{Z}_{p}$ has order $q$, let $H_{q}$ be the nonnormal subgroup $H_{q}=$ $\langle(a, 0)\rangle$ of size $q$. Then $H_{q}$ consists of the elements of the form $\left(a^{t}, 0\right)$, and its conjugates $H_{q}^{b}=(1, b) \cdot H_{q} \cdot(1,-b)$ consist of the elements of the form $\left(a^{t},\left(1-a^{t}\right) b\right)$. Geometrically, these are the subsets of $H$ and $H^{b}$, respectively, consisting of lines whose slope is a power of $a$.

Construction of the representations of $A_{p}$ requires that we fix a generator $\gamma$ of $\mathbb{Z}_{p}^{*}$. Define $\log : \mathbb{Z}_{p}^{*} \rightarrow \mathbb{Z}_{p-1}$ to be the isomorphism $\log \gamma^{t}=t$. Let $\omega_{p}$ denote the $p$ th root of unity $\mathrm{e}^{2 \pi i / p}$. Then $A_{p}$ has $p-1$ one-dimensional representations $\sigma_{s}$, namely the representations of $\mathbb{Z}_{p}^{*} \cong \mathbb{Z}_{p-1}$ given by $\sigma_{t}((a, b))=\omega_{p-1}^{t \log a}$. In addition, $A_{p}$ has one $(p-1)$-dimensional representation $\rho$ given by

$$
\rho((a, b))_{j, k}= \begin{cases}\omega_{p}^{b j}, & k=a j \bmod p, \quad 1 \leq j, k<p \\ 0 & \text { otherwise }\end{cases}
$$


where the indices $i$ and $j$ are elements of $\mathbb{Z}_{p}^{*}$. See [24, section 8.2] for a more detailed discussion.

Similarly, given a prime $p$ and a divisor $q$ of $p-1$, we consider the $q$-hedral groups, namely semidirect products $\mathbb{Z}_{q} \ltimes \mathbb{Z}_{p}$. These embed in $A_{p}$ in a natural way, namely, as the normal subgroups $N_{q}$ defined above. The dihedral groups are the special case where $q=2$.

The representations of $\mathbb{Z}_{q} \ltimes \mathbb{Z}_{p}$ include the $q$ one-dimensional representations of $\mathbb{Z}_{q}$ given by $\sigma_{\ell}\left(\left(a^{t}, b\right)\right)=\omega_{q}^{\ell t}$ for $\ell \in \mathbb{Z}_{q}$ and the $(p-1) / q$ distinct $q$-dimensional representations $\rho_{k}$ given by

$$
\rho_{k}\left(\left(a^{u}, b\right)\right)_{s, t}= \begin{cases}\omega_{p}^{k a^{s} b}, & t=s+u \bmod q \\ 0 & \text { otherwise }\end{cases}
$$

for each $0 \leq s, t<q$. Here $k$ ranges over the elements of $\mathbb{Z}_{p}^{*} / \mathbb{Z}_{q}$, or, to put it differently, $k$ takes values in $\mathbb{Z}_{p}^{*}$, but $\rho_{k}$ and $\rho_{k^{\prime}}$ are isomorphic if $k$ and $k^{\prime}$ are in the same coset of $\langle a\rangle$.

The representations of the affine and $q$-hedral groups are related as follows. The restriction of the $(p-1)$-dimensional representation $\rho$ of $A_{p}$ to $N_{q}$ is reducible and is isomorphic to the direct product of the $\rho_{k}$. Moreover, if we measure $\rho$ in a Gel'fandTsetlin basis such as (2) which is adapted to the tower of subgroups

$$
A_{p}>N_{q}>\{1\}
$$

then $\rho$ becomes block-diagonal, with $(p-1) / q$ blocks of size $q$, and these blocks are exactly the representations $\rho_{k}$ of $N_{q}$. (See [20] for an introduction to adapted bases and their uses in quantum computation.) We will use this fact in sections 4 and 5 below.

The affine and $q$-hedral groups are metacyclic groups, i.e., extensions of a cyclic group $\mathbb{Z}_{p}$ by a cyclic group $\mathbb{Z}_{q}$. In [14], Høyer shows how to perform the nonabelian Fourier transform over such groups (up to an overall phase factor) with a polynomial, i.e., polylog $(p)$, number of elementary quantum operations.

3. Full reconstructibility. In this section we show that conjugates of sufficiently large subgroups of the affine groups are fully reconstructible in polynomial time. For some values of $p$ and $q$, this allows us to completely solve the HSP for the $q$-hedral group $\mathbb{Z}_{q} \ltimes \mathbb{Z}_{p}$.

THEOREM 1. Let $p$ be prime and let $q$ be a divisor of $p-1$ for which $(p-1) / q=$ $\operatorname{poly} \log (p)$. Then the hidden conjugates $H_{q}^{b}$ of $H_{q}$ in $A_{p}$ are fully reconstructible.

Proof. First, consider the maximal nonnormal subgroup $H=\langle(\gamma, 0)\rangle$, where $\gamma$ is a generator of $\mathbb{Z}_{p}^{*}$. Carrying out Steps 1 through 3 of the Fourier sampling procedure outlined in the introduction results in a state $\psi_{3}$ over the group $G$ which is uniformly supported on a random left coset of the conjugate $H^{b}$. Using the procedure of [14], we now compute the quantum Fourier transform of this state over $A_{p}$ in the basis (2). The associated projection operator is

$$
\pi_{H^{b}}(\rho)_{j, k}=\frac{1}{p-1} \omega_{p}^{b(j-k)}
$$

for $1 \leq j, k<p$. This is a circulant matrix of rank 1 . More specifically, every column is some root of unity times the vector

$$
\left(u_{b}\right)_{j}=\frac{1}{p-1} \omega_{p}^{b j}
$$

Copyright $@$ by SIAM. Unauthorized reproduction of this article is prohibited. 
$1 \leq j<p$. This is also true of $\rho(c) \cdot \pi_{H^{b}}(\rho)$; since $\rho(c)$ has one nonzero entry per column, left multiplying by $\rho(c)$ simply multiplies each column of $\pi_{H^{b}}(\rho)$ by a phase. Note that in this case

$$
n_{\rho}=d_{\rho}|H| /|G|=(p-1) / p=1-1 / p,
$$

and so by $(1)$ we observe the $(p-1)$-dimensional representation $\rho$ with overwhelming probability $1-1 / p$.

Assuming that we observe $\rho$, we perform another change of basis: namely, we Fourier transform each column by left multiplying $\rho(\mathrm{cH})$ by the unitary matrix

$$
Q_{\ell, j}=\frac{1}{\sqrt{p-1}} \omega_{p-1}^{-\ell j}
$$

In terms of quantum operations, we apply the quantum Fourier transform over $\mathbb{Z}_{p-1}$ to the row register, while leaving the column register unchanged. We can now infer $b$ by measuring the frequency $\ell$. Specifically, we observe a given value of $\ell$ with probability

$$
P(\ell)=\left|\frac{1}{p-1} \sum_{j=1}^{p-1} \omega_{p}^{b j} \omega_{p-1}^{-\ell j}\right|^{2}=\frac{1}{(p-1)^{2}}\left|\sum_{j=1}^{p-1} \mathrm{e}^{2 i \theta j}\right|^{2}=\frac{1}{(p-1)^{2}} \frac{\sin ^{2}(p-1) \theta}{\sin ^{2} \theta}
$$

where

$$
\theta=\left(\frac{b}{p}-\frac{\ell}{p-1}\right) \pi
$$

Now note that for any $b$ there is an $\ell$ such that $|\theta| \leq \pi /(2(p-1))$. Since

$$
(2 x / \pi)^{2} \leq \sin ^{2} x \leq x^{2}
$$

for $|x| \leq \pi / 2$, this gives $P(\ell) \geq(2 / \pi)^{2}$.

Recall that the probability that we observed the $(p-1)$-dimensional representation $\rho$ in the first place is $n_{\rho}=1-1 / p$. Thus if we measure $\rho$, the column, and then $\ell$ and then guess that $b$ minimizes $|\theta|$, we will be correct with constant probability. This can be boosted to high probability, i.e., $1-o(1)$, by repeating the experiment a polynomial number of times. Alternately, we can use a POVM rather than the von Neumann measurement presented here and obtain the correct value of $b$ with high probability in a single measurement [1].

Now consider the more general case, where the hidden subgroup is a conjugate of the subgroup $H_{q}$ of order $q$. For convenience, let $\langle(a, 0)\rangle$ be a generator for $H_{q}$; then a given conjugate $H_{q}^{b}$ consists of the elements of the form $\left(a^{t},\left(1-a^{t}\right) b\right)$. We have

$$
\pi_{H_{q}^{b}}(\rho)_{j, k}=\frac{1}{q} \begin{cases}\omega_{p}^{b(j-k)}, & k=a^{t} j \text { for some } t \\ 0 & \text { otherwise }\end{cases}
$$

for $1 \leq j, k<p$. In other words, the nonzero entries are those for which $j$ and $k$ lie in the same coset of $\langle a\rangle \subset \mathbb{Z}_{p}^{*}$. The rank of this projection operator is thus the number of cosets equal to the index $(p-1) / q$ of $\langle a\rangle$ in $\mathbb{Z}_{p}^{*}$. Since $n_{\rho}$ is now $q / p$, we again observe $\rho$ with probability

$$
n_{\rho} \mathbf{r k} \pi_{H_{q}}(\rho)=(p-1) / p=1-1 / p .
$$


Following the same procedure as before, we carry out a partial measurement on the columns of $\rho$ and then Fourier transform the rows. After changing the variable of summation from $t$ to $-t$ and adding a phase shift of $\mathrm{e}^{-i \theta(p-1)}$ inside the $|\cdot|^{2}$, we obtain the probability that we observe a frequency $\ell$ conditional on finding ourselves in the $k$ th column:

$$
\begin{aligned}
P(\ell) & =\left|\frac{1}{\sqrt{q(p-1)}} \sum_{t=0}^{q-1} \omega_{p}^{b\left(a^{t} k \bmod p\right)} \omega_{p-1}^{-\ell\left(a^{t} k \bmod p\right)}\right|^{2} \\
& =\frac{1}{q(p-1)}\left|\sum_{t=0}^{q-1} \mathrm{e}^{2 i \theta\left(a^{t} k \bmod p\right)}\right|^{2} .
\end{aligned}
$$

Now note that the terms in the sum are of the form $\mathrm{e}^{i \phi}$, where (assuming without loss of generality that $\theta$ is positive)

$$
\phi \in[-\theta(p-1), \theta(p-1)] .
$$

If we again take $\ell$ so that $|\theta| \leq \pi /(2(p-1))$, then $\phi \in[-\pi / 2, \pi / 2]$ and all the terms in the sum have nonnegative real parts. We will obtain a lower bound on the real part of the sum by showing that a constant fraction of the terms have $\phi \in(-\pi / 3, \pi / 3)$ and thus have real part more than $1 / 2$. This is the case whenever $a^{t} k \in(p / 6,5 p / 6)$, and so it is sufficient to prove the following lemma.

Lemma 1. Let a have order $q=p / \operatorname{polylog}(p)$ in $\mathbb{Z}_{p}^{*}$, where $p$ is prime. Then at least $(1 / 3-o(1)) q$ of the elements in the coset $\langle a\rangle k$ are in the interval $(p / 6,5 p / 6)$.

Proof. We will prove this using Gauss sums, which quantify the interplay between the characters of $\mathbb{Z}_{p}$ and the characters of $\mathbb{Z}_{p}^{*}$. In particular, Gauss sums establish bounds on the distribution of powers of $a$. Specifically, if $a$ has order $q$ in $\mathbb{Z}_{p}^{*}$, then for any integer $k \not \equiv 0 \bmod p$ we have

$$
\sum_{t=0}^{q-1} \omega_{p}^{a^{t} k}=O\left(p^{1 / 2}\right)=o(p) .
$$

(See [17] and Appendix A.)

Now suppose $s$ of the elements $x$ in $\langle a\rangle k$ are in the set $(p / 6,5 p / 6)$, for which $\operatorname{Re} \omega_{p}^{x} \geq-1$, and the other $q-s$ elements are in $[0, p / 6] \cup[5 p / 6, p)$, for which $\operatorname{Re} \omega_{p}^{x} \geq$ $1 / 2$. Thus we have

$$
\operatorname{Re} \sum_{t=0}^{q-1} \omega_{p}^{a^{t} k} \geq(q / 2)-(3 s / 2) .
$$

If $s \leq(1 / 3-\epsilon) q$ for any $\epsilon>0$, this is $\Theta(q)$, a contradiction.

Now that we know that a fraction $1 / 3-\epsilon$ of the terms in (4) have real part at least $1 / 2$ and the others have real part at least 0 , we can take $\epsilon=1 / 12$ (say) and write

$$
P(\ell) \geq \frac{1}{q(p-1)}\left(\frac{q}{8}\right)^{2}=\frac{1}{64} \frac{q}{p-1}=\frac{1}{\operatorname{polylog}(p)} .
$$

Thus we observe the correct frequency with polynomially small probability, and this can be boosted to high probability by a polynomial number of repetitions. 
As we will now show, Theorem 1 implies that we can completely solve the HSP for certain $q$-hedral groups.

THEOREM 2. Let $p$ and $q$ be prime with $q=(p-1) / \operatorname{polylog}(p)$. Then subgroups of the $q$-hedral group $\mathbb{Z}_{q} \ltimes \mathbb{Z}_{p}$ are fully reconstructible.

Proof. First, note that we can fully reconstruct $H$ if it is nontrivial and normal. We do this by reconstructing the normal core of $H$,

$$
C(H)=\bigcap_{\gamma \in G} \gamma H \gamma^{-1}
$$

using the techniques of [13] (the weak standard method). The $q$-hedral groups have the special property that nonnormal subgroups contain no nontrivial normal subgroups; in particular, if $H$ is nonnormal, then $C(H)$ is the trivial subgroup. Thus by reconstructing $C(H)$, we either learn that $H=C(H)$ or learn that $H$ is either trivial or nonnormal. Furthermore, if $H$ is trivial, we will learn this by checking our reconstruction against the oracle $f$ and finding that it is incorrect. Therefore, it suffices to consider the nonnormal subgroups.

If $q$ is prime, then the nonnormal subgroups of $\mathbb{Z}_{q} \ltimes \mathbb{Z}_{p}$ are all conjugate to a single subgroup $K \cong \mathbb{Z}_{q}$, as any such subgroup has the form $\left\{(a,(1-a) z) \mid a \in \mathbb{Z}_{q}<\right.$ $\left.\operatorname{Aut}\left(\mathbb{Z}_{p}\right) \cong \mathbb{Z}_{p}^{*}\right\}$; in this case the HSP reduces to the HCP for $K$. While one can construct a proof similar to that of Theorem 1 directly for the $q$-hedral groups, it is convenient to embed them in $A_{p}$ using the isomorphisms $N_{q} \cong \mathbb{Z}_{q} \ltimes \mathbb{Z}_{p}$ and $H_{q} \cong K$ and appeal to Theorem 1.

Now suppose we have an oracle $f: \mathbb{Z}_{q} \times \mathbb{Z}_{p} \rightarrow S$. We extend this to an oracle $f^{\prime}$ on $A_{p}$ as follows. Choose a generator $\gamma \in \mathbb{Z}_{p}^{*}$ and one of the $q-1$ elements $a \in \mathbb{Z}_{p}^{*}$ of order $q$, and let

$$
f^{\prime}: A_{p} \rightarrow S \times\langle a\rangle
$$

where

$$
f^{\prime}((a, b))=\left(f\left(\left(\left\lfloor\frac{\log a}{(p-1) / q}\right\rfloor, b\right)\right), a^{q}\right),
$$

recalling that $\log \gamma^{t}=t$. The second component of $f^{\prime}$ serves to distinguish the cosets of $N_{q}$ from each other, while the first component maps each coset of $N_{q}$ to $\mathbb{Z}_{q} \ltimes \mathbb{Z}_{p}$ with the element of $\mathbb{Z}_{q}$ written additively, rather than multiplicatively. (This last step is not strictly necessary - after all, we could have written the elements of $A_{p}$ in additive form in the first place - but it can be carried out with Shor's algorithm for the discrete logarithm [25].) This reduces the HCP for $K$ (and therefore the HSP) on $\mathbb{Z}_{q} \ltimes \mathbb{Z}_{p}$ to the HCP for $H_{q}$ on $A_{p}$, completing the proof.

As an example of Theorem 2, if $q$ is a Sophie Germain prime, i.e., one for which $p=2 q+1$ is also a prime, we can completely solve the HSP for $\mathbb{Z}_{q} \ltimes \mathbb{Z}_{p}$.

4. Information-theoretic reconstructibility. In this section, we show that all subgroups of the affine and $q$-hedral groups, regardless of their size, are informationtheoretically reconstructible. We start by considering the HCP for subgroups $H_{q}$ in $A_{p}$. Then in Theorem 5 we show that we can identify the conjugacy class of a hidden subgroup and therefore the subgroup itself. This generalizes the results of Ettinger and Høyer [6], who show information-theoretic reconstructibility for the dihedral groups, i.e., the case $q=2$. 
TheOREM 3. Let $p$ be prime and let $q$ divide $p-1$. Then the hidden conjugates of $H_{q}$ in $A_{p}$ are information-theoretically reconstructible.

Proof. Suppose $a \in \mathbb{Z}_{p}^{*}$ has order $q$. Recall that $H_{q}$ and its conjugates $H_{q}^{b}$ are maximal in the subgroup $N_{q} \cong \mathbb{Z}_{q} \ltimes \mathbb{Z}_{p}$. We wish to show that there is a measurement whose outcomes, given two distinct values of $b$, have large (i.e., $1 / \operatorname{polylog}(p)$ ) total variation distance. First, we perform a series of partial measurements as follows:

(i) Measure the name of the representation of $A_{p}$. If this is not $\rho$, try again. Otherwise, continue.

(ii) Measure the name of the representation $\rho_{k}$ of $N_{q}$ inside $\rho$.

(iii) Measure the column of $\rho_{k}$.

(iv) Perform a POVM with $q$ outcomes, in each of which the row $s \in \mathbb{Z}_{q}$ is $u$ or $(u+1) \bmod q$.

As in Theorem 1, we measure the (p-1)-dimensional representation of $A_{p}$ in a chosen basis. Recall that in the adapted basis (2) the restriction of $\rho$ to $N_{q}$ is block-diagonal, where the $(p-1) / q$ blocks are the $q$-dimensional representations $\rho_{k}$ of $N_{q}$. Therefore, the projection operator $\pi_{H_{q}^{b}}(\rho)$ is block-diagonal, and each of its blocks is one of the projection operators $\pi_{H_{q}^{b}}\left(\rho_{k}\right)$. Summing $\rho_{k}$ over $H_{q}^{b}=\left\{\left(a^{t},\left(1-a^{t}\right) b\right)\right\}$ gives

$$
\left(\pi_{H_{q}^{b}}\left(\rho_{k}\right)\right)_{s, t}=\frac{1}{q} \omega_{p}^{k\left(a^{s}-a^{t}\right) b}
$$

for $0 \leq s, t<q$. This is a matrix of rank 1 , where each column (even after left multiplication by $\left.\rho_{k}(c)\right)$ is some root of unity times the vector $\left(u_{k}\right)_{s}=(1 / q) \omega_{p}^{k a^{s} b}$. Since $n_{\rho}=q / p$, the probability that we observe a particular $\rho_{k}$ is $q / p$. Since $\pi_{H_{q}^{b}}(\rho)$ has $(p-1) / q$ blocks of this kind, it has rank $(p-1) / q$, and the total probability that we observe $\rho$ is $(p-1) / p=1-1 / p$ as before.

Then these four partial measurements determine $k$, remove the effect of the coset, and determine that the row has one of two values, $u$ or $u+1$. Up to an overall phase we can write this as a two-dimensional vector:

$$
\frac{1}{\sqrt{2}}\left(\begin{array}{c}
\omega_{p}^{k a^{u} b} \\
\omega_{p}^{k a^{u+1} b}
\end{array}\right) .
$$

Our only goal in doing this is to create a one-qubit state where the relative phase between the two basis vectors depends on the conjugate $b$. Moreover, the relative phase is multiplied by the irrep label $k$, which is uniformly random. As a result, the typical angle between the states corresponding to any two distinct cosets $b, b^{\prime}$ will be $\Omega(1)$, and a simple measurement yields a constant variation distance between them.

To make this precise, apply the Hadamard transform

$$
\frac{1}{\sqrt{2}}\left(\begin{array}{rr}
1 & 1 \\
1 & -1
\end{array}\right)
$$

and measure according to the resulting basis. The probability that we observe the first or second basis vector is then $\cos ^{2} \theta$ and $\sin ^{2} \theta$, respectively, where $\theta=\left(k a^{u}(a-\right.$ 1) $b \pi) / p$. Now when we observe a $q$-dimensional representation $\rho_{k}$ of $N_{k}$, the observed label $k$ is uniformly distributed over $\mathbb{Z}_{p}^{*} / \mathbb{Z}_{q}$. Moreover, when we perform the POVM in step (iv) above, the $u$ we observe is uniformly distributed over $\mathbb{Z}_{q}$. It follows that the coefficient $m=k a^{u}(u-1)$ is uniformly distributed over $\mathbb{Z}_{p}^{*}$. For any two distinct $b, b^{\prime}$, the total variation distance is then

$$
\frac{1}{2(p-1)} \sum_{m \in \mathbb{Z}_{p}^{*}}\left(\left|\cos ^{2} \frac{\pi m b}{p}-\cos ^{2} \frac{\pi m b^{\prime}}{p}\right|+\left|\sin ^{2} \frac{\pi m b}{p}-\sin ^{2} \frac{\pi m b^{\prime}}{p}\right|\right) .
$$


This we rewrite

$$
\begin{aligned}
& \frac{1}{p-1} \sum_{m \in \mathbb{Z}_{p}^{*}}\left|\cos ^{2} \frac{\pi m b}{p}-\cos ^{2} \frac{\pi m b^{\prime}}{p}\right| \\
= & \frac{1}{2(p-1)} \sum_{m \in \mathbb{Z}_{p}}\left|\cos \frac{2 \pi m b}{p}-\cos \frac{2 \pi m b^{\prime}}{p}\right| \\
\geq & \frac{1}{4(p-1)} \sum_{m \in \mathbb{Z}_{p}}\left(\cos \frac{2 \pi m b}{p}-\cos \frac{2 \pi m b^{\prime}}{p}\right)^{2} \\
= & \frac{p}{4(p-1)}>\frac{1}{4} .
\end{aligned}
$$

(Adding the $m=0$ term contributes zero to the sum in the second line. In the third line we use the facts that $|x| \leq x^{2} / 2$ for all $|x| \leq 2$, the average of $\cos ^{2} x$ is $1 / 2$, and the two cosines have zero inner product.)

Since the total variation distance between any two distinct conjugates is bounded below by a constant, we can distinguish between the $p$ different conjugates with only $O(\log p)=\operatorname{poly}(n)$ samples. Thus, hidden conjugates in $A_{p}$ are informationtheoretically reconstructible, completing the proof.

By embedding the $q$-hedral groups in $A_{p}$ as in Theorem 2, we can generalize Theorem 3 to the $q$-hedral groups ( $q$ not necessarily prime) as follows. Let $p$ be prime, let $q$ be a divisor of $p-1$, and let $q^{\prime}$ be a divisor of $q$. The $q$-hedral group $\mathbb{Z}_{q} \ltimes \mathbb{Z}_{p}$ has a unique normal subgroup $K \cong \mathbb{Z}_{q^{\prime}} \ltimes \mathbb{Z}_{p}$, in which there is a maximal subgroup $H_{q^{\prime}}$ of order $q^{\prime}$. Moreover, all nonnormal subgroups of order $q^{\prime}$ are conjugates of $H_{q^{\prime}}$.

THEOREM 4. If $p, q, q^{\prime}$, and $H_{q^{\prime}}$ are as above, then the hidden conjugates of $H_{q^{\prime}}$ in $\mathbb{Z}_{q} \ltimes \mathbb{Z}_{p}$ are information-theoretically reconstructible.

We now wish to information-theoretically reconstruct all subgroups of the affine and $q$-hedral groups. We can do this by using the fact that, except for normal subgroups, there is a unique conjugacy class of each order $q^{\prime}$, namely the conjugates of $H_{q^{\prime}}$. Thus if we can determine the order of $H$, this determines its conjugacy class, and we can information-theoretically reconstruct which conjugate it is using, Theorem 3 or Theorem 4.

THEOREM 5. Subgroups of the q-hedral groups $\mathbb{Z}_{q} \ltimes \mathbb{Z}_{p}$, including the affine groups $A_{p}$, are information-theoretically reconstructible.

Proof. As in Theorem 2, we can (fully) reconstruct normal subgroups, and so it suffices to consider nonnormal subgroups $H$. As discussed above, if we can determine $|H|=q^{\prime}$, then we know that it is one of the conjugates of $H_{q^{\prime}}$, and we can reconstruct it information-theoretically using Theorem 3 or Theorem 4.

Now let the oracle be $f: \mathbb{Z}_{q} \ltimes \mathbb{Z}_{p} \rightarrow S$, and let $p_{1}^{\alpha_{1}} \ldots p_{k}^{\alpha_{k}}$ be the prime factorization of $q$, in which case $k \leq \sum_{i} \alpha_{i}=O(\log q)$. For each $i \in\{1, \ldots, k\}$ and each $\alpha \in\left\{0, \ldots, \alpha_{i}\right\}$, we will determine if $p_{i}^{\alpha}|| H \mid$, and taking the largest such $\alpha$ for each $i$ gives the prime factorization of $|H|$.

To do this, for each $i \in[k]$ and $1 \leq \alpha \leq \alpha_{i}$, let $\Upsilon_{i}^{\alpha}: \mathbb{Z}_{q} \ltimes \mathbb{Z}_{p} \rightarrow \mathbb{Z}_{q / p_{i}^{\alpha}}$ be the homomorphism given by

$$
\Upsilon_{i}^{\alpha}:(a, b) \mapsto a^{p_{i}^{\alpha}}
$$


Then let

$$
A_{i}^{\alpha_{i}}=\operatorname{ker} \Upsilon_{i}^{\alpha}=\left\{\gamma \in \mathbb{Z}_{q} \ltimes \mathbb{Z}_{p} \mid \gamma^{p_{i}^{\alpha_{i}}}=\mathbf{1}\right\},
$$

where 1 denotes the identity element of $\mathbb{Z}_{q} \ltimes \mathbb{Z}_{p}$. $A_{i}^{\alpha_{i}}$ is the subgroup of $\mathbb{Z}_{q} \ltimes \mathbb{Z}_{p}$ consisting of all elements whose orders are a multiple of $p_{i}^{\alpha}$. Now consider the function

$$
f^{\prime}: \mathbb{Z}_{q} \ltimes \mathbb{Z}_{p} \rightarrow S \times \mathbb{Z}_{q / p_{i}^{\alpha}}
$$

given by

$$
f^{\prime}(\gamma)=\left(f(\gamma), \Upsilon_{i}^{\alpha}(\gamma)\right)
$$

Observe that $f^{\prime}$ is constant (and distinct) on the left cosets of $H \cap A_{i}^{\alpha}$ and, furthermore, the subgroup $H \cap A_{i}^{\alpha}$ has order $p^{\alpha}$ if and only if $p^{\alpha}$ divides $|H|$. We may then determine if $H \cap A_{i}^{\alpha}$ has order $p^{\alpha}$ by assuming that it does, reconstructing $H$ with Theorem 4 using $f^{\prime}$ as the oracle, and checking the result against the original oracle $f$. This allows us to determine the prime factorization of $|H|$ as desired, and the theorem follows.

As in the dihedral case [6], we know of no polynomial-time algorithm which can reconstruct the most likely $b$ from these queries. However, Kuperberg [18] gives a quantum algorithm for the HSP in the dihedral group, and more generally the hidden shift problem, that runs in subexponential $\left(\mathrm{e}^{O\left(\log ^{1 / 2} p\right)}\right)$ time. Since we can reduce the HSP on $\mathbb{Z}_{q} \ltimes \mathbb{Z}_{p}$ to a hidden shift problem by focusing on two cosets of $\mathbb{Z}_{p}$, this algorithm applies to the $q$-hedral groups as well.

5. Random vs. adapted bases. In Theorems 3 and 5, we measured the highdimensional representation $\rho$ in a specific basis which is adapted to the subgroup structure of $A_{p}$ and the $q$-hedral groups. In contrast, we show in this section that if we measure $\rho$ in a random basis instead, then for all but the largest values of $q$ we need an exponential number of measurements in order to information-theoretically distinguish conjugate subgroups from each other.

THEOREM 6. Let $p$ be prime and let $q$ be a divisor of $p-1$ for which $q<p^{1-\epsilon}$ for some $\epsilon>0$. Let $P_{b}(v)$ be the probability that we observe a basis vector $v$ in the Fourier basis if the hidden subgroup is $H_{q}^{b}$. If we measure $\rho$ in a random basis, then for any two $b, b^{\prime}$, with high probability the $\ell_{1}$ distance between these probability distributions is exponentially small. In particular, there exists $\beta>0$, depending only on $\epsilon$, such that

$$
\sum_{v}\left|P_{b}(v)-P_{b^{\prime}}(v)\right|<p^{-\beta}
$$

Thus it takes an exponentially large number of measurements to distinguish the conjugates $H_{q}^{b}$ and $H_{q}^{b^{\prime}}$.

Proof. Since we observe the high-dimensional representation $\rho$ with probability $1-1 / p$, it suffices to consider the $\ell_{1}$ distance summed over the $d_{\rho}=p-1$ basis vectors of $\rho$. In fact, we will show that $P_{b}(v)$ is exponentially close to the uniform distribution for all $b$.

Write $\pi=\pi_{H_{q}^{b}}(\rho)$. Then the probability that we observe a given basis vector $v$, conditioned on observing $\rho$, is

$$
P_{b}(v)=\frac{1}{\mathbf{r k} \pi}|\pi \cdot v|^{2}
$$

Copyright (C) by SIAM. Unauthorized reproduction of this article is prohibited. 
If $v$ is uniformly random with norm 1 , the expectation of $|\pi \cdot v|_{2}^{2}$ is $(\mathbf{r k} \pi) / d_{\rho}$, and so the expectation of $P_{b}(v)$ is $1 / d_{\rho}$. We will use the following lemma to show that when rk $\pi$ is sufficiently large, $P_{b}(v)$ is tightly concentrated around this expectation.

Lemma 2. Let $\pi$ be a projection operator of rank $r$ in a d-dimensional space, and let $v$ be a random d-dimensional vector of unit length. Then for all $0<\delta<2$,

$$
\operatorname{Pr}\left[\left.|| \pi \cdot v\right|_{2} ^{2}-\frac{r}{d} \mid>\delta \frac{r}{d}\right]<4 \mathrm{e}^{-r \delta^{2} / 48} .
$$

Proof. We use an argument similar to [10]. We can think of a random $d$ dimensional complex vector $v$ as a random $2 d$-dimensional real vector of the same length, and we can think of this in turn as

$$
v_{i}=\frac{w_{i}}{\sum_{i=1}^{2 d} w_{i}^{2}}
$$

where the $w_{i}$ are independent Gaussian variables with zero mean and unit variance. By choosing a basis in which $\pi$ projects onto the first $r$ (complex) components of $v$, we have

$$
|\pi \cdot v|_{2}^{2}=\frac{\sum_{i=1}^{2 r} w_{i}^{2}}{\sum_{i=1}^{2 d} w_{i}^{2}}=\frac{r}{d} \frac{(1 / 2 r) \sum_{i=1}^{2 r} w_{i}^{2}}{(1 / 2 d) \sum_{i=1}^{2 d} w_{i}^{2}} .
$$

Now we use the following Chernoff bound, which can be derived from the moment generating function. For any $t$, we have

$$
\operatorname{Pr}\left[\left|\left(\frac{1}{t} \sum_{i=1}^{t} w_{i}^{2}\right)-1\right|>\epsilon\right]<2\left[(1+\epsilon)^{1 / 2} \mathrm{e}^{-\epsilon / 2}\right]^{t} .
$$

For $|\epsilon|<1 / 2$, we have $\ln (1+\epsilon)<\epsilon-\epsilon^{2} / 3$, and this becomes

$$
\operatorname{Pr}\left[\left|\left(\frac{1}{t} \sum_{i=1}^{t} w_{i}^{2}\right)-1\right|>\epsilon\right]<2 \mathrm{e}^{-t \epsilon^{2} / 6} .
$$

Now, for any $a, b$, if $|a / b-1|>\delta$, where $\delta<2$, then either $|a-1|>\delta / 4$ or $|b-1|>\delta / 4$. Taking the union bound over these events where $a=(1 / 2 r) \sum_{i=1}^{2 r} w_{i}^{2}$ and $b=(1 / 2 d) \sum_{i=1}^{2 d} w_{i}^{2}$, setting $\epsilon=\delta / 4$ and $t=2 r \leq 2 d$ in (5) gives the stated bound.

Setting $d=d_{\rho}$ and $r=\mathbf{r k} \pi$, Lemma 2 and the union bound imply that, for any constant $A>\sqrt{48}$, if

$$
\delta=A \sqrt{\frac{\log d_{\rho}}{\mathrm{rk} \pi}}
$$

then, with high probability, for all $d_{\rho}$ basis vectors $v$ we have

$$
\left|P_{b}(v)-\frac{1}{d_{\rho}}\right|<\frac{\delta}{d_{\rho}}
$$

Summing over all $v$, this implies that the $\ell_{1}$ distance between $P_{b}(v)$ and the uniform 
distribution is at most $\delta$. Now recall that rk $\pi=(p-1) / q$. If $q<p^{1-\epsilon}$, then rk $\pi>p^{\epsilon}$, and (6) gives $\delta<p^{-\beta}$, where $\beta=\epsilon / 3$, say. Since $P_{b}(v)$ is within $\delta$ of the uniform distribution for all $b$, doubling the constant $A$ and using the triangle inequality completes the proof.

Several remarks are in order. First, just as for the dihedral group, we can information-theoretically distinguish conjugate subgroups if we use a random basis within each $q$-dimensional block of $\rho$. The problem is that rather than having this block-diagonal structure, a random basis cuts across these blocks, mixing different "frequencies" $\rho_{k}$ and canceling out the useful information. To be more precise, a random basis is not adapted to the subgroup structure of $A_{p}$; it does not "know" that $\rho$ decomposes into a direct sum of the $\rho_{k}$ when restricted to $N_{q}$.

Second, it is worth noting that for the values of $q$ for which we have an algorithm for full (as opposed to information-theoretic) reconstruction, namely $q=p / \operatorname{polylog}(p)$, a random basis works as well, since the $\ell_{1}$ distance $\delta$ becomes $1 / \operatorname{polylog}(p)$. Based on the strong evidence from representation theory that some bases are much better for computation than others, we conjecture that, for some families of groups, adapted bases allow full reconstruction while random bases do not; but this remains an open question.

Third, while we focused above on distinguishing conjugate subgroups from each other, in fact our proof shows that if $q<p^{1-\epsilon}$, a random basis is incapable of distinguishing $H_{q}$ from the trivial subgroup. In contrast, Theorems 3 and 5 show that an adapted basis allows us to do this.

6. Failure of the abelian Fourier transform. In [6] the abelian Fourier transform over $\mathbb{Z}_{2} \times \mathbb{Z}_{p}$ is used in a reconstruction algorithm for the dihedral groups. Using this sort of "forgetful" abelian Fourier analysis it is similarly information-theoretically possible to reconstruct subgroups of the $q$-hedral groups when $q$ is small enough.

However, it does not seem possible to reconstruct subgroups of $A_{p}$ using the abelian Fourier transform. In particular, we show in this section that if we think of the affine group as a direct product $\mathbb{Z}_{p}^{*} \times \mathbb{Z}_{p}$ rather than a semidirect product, then the conjugates of the maximal subgroup become indistinguishable. This is not surprising, since in an abelian group conjugates are identical by definition, but it helps illustrate that nonabelian HSPs require nonabelian approaches (most naturally, in our view, representation theory).

Let us consider the HCP for the maximal subgroup $H=\langle(\gamma, 0)\rangle$, where $\gamma$ is a generator of $\mathbb{Z}_{p}^{*}$. In that case, the characters of $\mathbb{Z}_{p}^{*} \times \mathbb{Z}_{p}$ are simply $\rho_{k, \ell}\left(\gamma^{t}, b\right)=$ $\omega_{p-1}^{k t} \omega_{p}^{\ell b}$. Summing these over $H_{q}=\left\{\left(z,(1-z) b \mid z \in \mathbb{Z}_{p}^{*}\right\}\right.$ shows that we observe the character $(k, \ell)$ with probability

$$
\begin{aligned}
P(k, \ell) & =\frac{1}{p(p-1)^{2}}\left|\sum_{t \in \mathbb{Z}_{p-1}} \omega_{p-1}^{k t} \omega_{p}^{\ell\left(1-\gamma^{t}\right) b}\right|^{2} \\
& =\frac{1}{p(p-1)^{2}}\left|\sum_{x \in \mathbb{Z}_{p}^{*}} \omega_{p-1}^{k \log _{\gamma} x} \omega_{p}^{-\ell x b}\right|^{2} .
\end{aligned}
$$

This is the inner product of a multiplicative character with an additive one, which is 
another Gauss sum. In particular, assuming $b \neq 0$, we have

$$
\begin{aligned}
P(0,0) & =1 / p, \\
P(0, \ell \neq 0) & =1 /\left(p(p-1)^{2}\right), \\
P(k \neq 0,0) & =0, \\
P(k \neq 0, \ell \neq 0) & =1 /(p-1)^{2}
\end{aligned}
$$

(see Appendix A). Since these probabilities do not depend on $b$, the different conjugates $H^{b}$ with $b \neq 0$ are indistinguishable from each other. Thus it appears essential to use the nonabelian Fourier transform and the high-dimensional representations of $A_{p}$.

7. Hidden shift problems. Using the natural action of the affine group on $\mathbb{Z}_{p}$, we can apply our algorithm for the HCP studied above to a natural family of hidden shift problems. Specifically, let $M$ be a multiplicative subgroup of $\mathbb{Z}_{p}^{*}$ of index $r>1$, let $S$ be some set of $r+1$ symbols, and let $f: \mathbb{Z}_{p} \rightarrow S$ be a function for which

$$
f(x)=f(m x) \Leftrightarrow m \in M
$$

for every $x \in \mathbb{Z}_{p}$. Observe that $f$ is constant on the (multiplicative) cosets of $M$ and takes distinct values on distinct cosets; to put it differently, $f(x)$ is an injective function of the multiplicative order of $x$ mod $r$. Furthermore, $f(0) \neq f(x)$ for any nonzero $x$. The hidden shift problem associated with $f$ is the problem of determining an unknown element $s \in \mathbb{Z}_{p}$ given oracle access to the shifted function

$$
f_{s}(x)=f(x-s) .
$$

Such functions have remarkable pseudorandom properties, and have been proposed as pseudorandom generators for cryptographic purposes, where $s$ acts as the seed to generate the sequence (see, e.g., [5]).

The special case when $f: \mathbb{Z}_{p} \rightarrow \mathbb{C}$ is a Legendre symbol, that is, a multiplicative character of $\mathbb{Z}_{p}^{*}$ extended to all of $\mathbb{Z}_{p}$ by setting $f(0)=0$, was studied by van Dam, Hallgren, and Ip [4]. They give efficient quantum algorithms for these hidden shift problems for all characters of $\mathbb{Z}_{p}^{*}$. Their algorithms, however, make explicit use of the complex values taken by the character, whereas the algorithms we present here depend only on the symmetries of the underlying function $f$; in particular, in our case $f$ can be an arbitrary injective function from a multiplicative character into a set $S$. On the other hand, their algorithms are efficient for characters of any order, while our algorithms require that $r$ be at most polylogarithmic in $p$.

Returning to the general problem defined above, let $\mathcal{F}\left(\mathbb{Z}_{p}, S\right)$ denote the collection of $S$-valued functions on $\mathbb{Z}_{p}$. Note that the affine group $A_{p}$ acts on the set $\mathcal{F}\left(\mathbb{Z}_{p}, S\right)$ by assigning $\alpha \cdot g(x)=g\left(\alpha^{-1}(x)\right)$ for each $\alpha \in A_{p}$ and $g \in F\left(\mathbb{Z}_{p}, S\right)$. In particular, $f_{s}=(1, s) \cdot f$.

Now note that the isotropy subgroup of $f$, namely the subgroup of $A_{p}$ that fixes the cosets of $M$, is precisely $H_{q}=\langle(a, 0)\rangle$, where $a \in \mathbb{Z}_{p}^{*}$ has order $q=(p-1) / r$. As we have $f_{s}=(1, s) \cdot f$, the isotropy subgroup of $f_{s}$ is the conjugate subgroup $H_{q}^{s}=(1, s) \cdot H_{q} \cdot(1,-s)$. Now observe that if we define $F_{s}: A_{p} \rightarrow\left(\mathbb{Z}_{p}\right)^{p}$ so that $F_{s}(\alpha)$ is the $p$-tuple $\left(\alpha f_{s}(0), \alpha f_{s}(1), \ldots, \alpha f_{s}(p-1)\right)$, then

$$
F_{s}(\alpha)=F_{s}(\beta) \Leftrightarrow \alpha^{-1} \beta \in H_{q}^{s} ;
$$


i.e., $F_{s}$ is constant precisely on the left cosets of $H_{q}^{s}$. Evidently, then, the solution to the HCP given by the oracle $F_{s}$ determines the solution to the hidden shift problem given by $f_{s}$. Unfortunately, the values of the oracle $F_{s}$ are of exponential sizewe cannot afford to evaluate $\alpha f_{s}(x)$ for all $x \in \mathbb{Z}_{p}$. The same symmetry expressed in (7), however, can be obtained efficiently by selecting an appropriate subset $R=$ $\left\{x_{1}, \ldots, x_{m}\right\} \subset \mathbb{Z}_{p}$ and considering the oracle that samples $\alpha f_{s}$ on $R$, that is,

$$
F_{s}^{R}(\alpha)=\left(\alpha f_{s}\left(x_{1}\right), \ldots, \alpha f_{s}\left(x_{m}\right)\right) \text {. }
$$

Of course, we have $\alpha f_{s}=\beta f_{s} \Rightarrow F_{s}^{R}(\alpha)=F_{s}^{R}(\beta)$ regardless of $R$; the difficulty is finding a small set $R$ for which $F_{s}^{R}(\alpha)=F_{s}^{R}(\beta) \Rightarrow \alpha f_{s}=\beta f_{s}$. We show below that a set of $O(\log p)$ elements selected uniformly at random from $\mathbb{Z}_{p}$ has this property with high probability.

Considering that $\alpha f_{s}(x)=\alpha \cdot(1, s) \cdot f(x)$, it suffices to show that if $\alpha f \neq \beta f$, then

$$
\operatorname{Pr}_{x}[\alpha f(x)=\beta f(x)] \leq 1 / 2,
$$

where $x$ is selected uniformly at random in $\mathbb{Z}_{p}$. Note that for affine functions $\alpha$ and $\beta$ and an element $x \in \mathbb{Z}_{p}$ for which $\beta^{-1}(x) \neq 0$,

$$
\alpha f(x)=\beta f(x) \Leftrightarrow \frac{\alpha^{-1}(x)}{\beta^{-1}(x)} \in M .
$$

The function $\alpha^{-1}(x) / \beta^{-1}(x)$ is a fractional linear transform, i.e., the ratio of two linear functions; such functions are the discrete analogues of the Möbius transformations in the complex plane. As in the complex case, the fractional linear transform $\gamma(x) / \delta(x)$ is a bijection on the projective space $\mathbb{Z}_{p} \cup\{\infty\}$ unless $\gamma$ and $\delta$ share a root, or, equivalently, there is a scalar $z \in \mathbb{Z}_{p}^{*}$ such that $\gamma(x)=z \delta(x)$. If $\alpha^{-1}(x) / \beta^{-1}(x)$ is injective, we can immediately conclude that

$$
\operatorname{Pr}_{x}[\alpha f(x)=\beta f(x)] \leq|M| /(p-1)=1 / r \leq 1 / 2 .
$$

Otherwise, $\alpha^{-1}(x) / \beta^{-1}(x)=z$ for some scalar $z$. Since $\alpha f \neq \beta f$, however, in this case we must have $z \in \mathbb{Z}_{p}^{*} \backslash M$. In particular, $f(z y) \neq f(y)$ for any $y \neq 0$, and so

$$
\operatorname{Pr}_{x}[\alpha f(x)=\beta f(x)]=1 / p,
$$

since this occurs only at the unique root $x$ of $\alpha^{-1}(x)=0$.

In either case, then, $\alpha f$ and $\beta f$ differ on at least half the elements of $\mathbb{Z}_{p}$ whenever $\alpha$ and $\beta$ belong to different cosets of $H_{q}^{s}$. It follows that if $R \subset \mathbb{Z}_{p}$ consists of $m$ elements chosen independently and uniformly at random from $\mathbb{Z}_{p}$, we have

$$
\operatorname{Pr}_{R}[\forall x \in R, \alpha f(x)=\beta f(x)] \leq 1 / 2^{m}
$$

for any $\alpha, \beta \in A_{p}$ with $\alpha^{-1} \beta \notin H_{q}$. Taking a union bound over all pairs of left cosets of $H_{q}$,

$$
\operatorname{Pr}_{R}\left[\exists \alpha, \beta \in A_{p}: \alpha^{-1} \beta \notin H_{q} \forall x \in R, \alpha f(x)=\beta f(x)\right] \leq\left(\frac{p(p-1)}{\left|H_{q}\right|}\right)^{2} \frac{1}{2^{m}} .
$$

Selecting $m=5 \log p$ ensures that this probability is less than $1 / p$.

Since we showed in section 3 that we can identify a hidden conjugate of $H_{q}$ whenever $H_{q}$ is of polylogarithmic index in $\mathbb{Z}_{p}^{*}$, this provides an efficient solution to the hidden shift problem so long as $p / q=\operatorname{polylog}(p)$.

Copyright $\odot$ by SIAM. Unauthorized reproduction of this article is prohibited. 
8. Closure under extending small groups. In this section we show that for any polynomial-size group $K$ and any $H$ for which we can solve the HSP, we can also solve the HSP for any extension of $K$ by $H$, i.e., any group $G$ with $K \triangleleft G$ and $G / K \cong H$. (Note that this is more general than split extensions, i.e., semidirect products $H \ltimes K$.) This includes the case discussed in [13] of Hamiltonian groups, since all such groups are direct products (and hence extensions) by abelian groups of the quaternion group $Q_{8}$ [23]. It also includes the case discussed in [8] of groups with commutator subgroups of polynomial size, such as extra-special $p$-groups, since in that case $K=G^{\prime}$ and $H \cong G / G^{\prime}$ is abelian. Indeed, our proof is an easy generalization of that in $[8]$.

THEOREM 7. Let $H$ be a group for which hidden subgroups are fully reconstructible and $K$ a group of polynomial size in $\log |H|$. Then hidden subgroups in any extension of $K$ by $H$, i.e., any group $G$ with $K \triangleleft G$ and $G / K \cong H$, are fully reconstructible.

Proof. We assume that $G$ and $K$ are encoded in such a way that multiplication can be carried out in classical polynomial time. We fix some transversal $t(h)$ of the left cosets of $K$. First, note that any subgroup $L \subseteq G$ can be described in terms of (i) its intersection $L \cap K$, (ii) its projection $L_{H}=L /(L \cap K) \subseteq H$, and (iii) a representative $\eta(h) \in L \cap(t(h) \cdot K)$ for each $h \in L_{H}$. Then each element of $L_{H}$ is associated with some left coset of $L \cap K$, i.e., $L=\bigcup_{h \in L_{H}} \eta(h) \cdot(L \cap K)$. Moreover, if $S$ is a set of generators for $L \cap K$ and $T$ is a set of generators for $L_{H}$, then $S \cup \eta(T)$ is a set of generators for $L$.

We can reconstruct $S$ in classical polynomial time simply by querying the function $h$ on all of $K$. Then $L \cap K$ is the set of all $k$ such that $f(k)=f(1)$, and we construct $S$ by adding elements of $L \cap K$ to it one at a time until they generate all of $L \cap K$.

To identify $L_{H}$, as in [8] we define a new function $f^{\prime}$ on $H$ consisting of the unordered collection of the values of $f$ on the corresponding left coset of $K$ :

$$
f^{\prime}(h)=\{f(g) \mid g \in t(h) \cdot K\} .
$$

Each query to $f^{\prime}$ consists of $|K|=\operatorname{poly}(n)$ queries to $f$. The level sets of $f^{\prime}$ are clearly the cosets of $L_{H}$, and so we reconstruct $L_{H}$ by solving the HSP on $H$. This yields a set $T$ of generators for $L_{H}$.

It remains to find a representative $\eta(h)$ in $L \cap(t(h) \cdot K)$ for each $h \in T$. We simply query $f(g)$ for all $g \in t(h) \cdot K$ and set $\eta(h)$ to any $g$ such that $f(g)=f(1)$. Since $|T|=O(\log |H|)=\operatorname{poly}(n)$, this can be done in polynomial time, completing the proof.

Unfortunately, we cannot iterate this construction more than a constant number of times, since doing so would require a superpolynomial number of queries to $f$ for each query of $f^{\prime}$. If $K$ has superpolynomial size, it is not clear how to obtain $\eta(h)$, even when $H$ has only two elements. Indeed, this is precisely the difficulty with the dihedral group.

9. Conclusion and directions for further work. We have shown that the "strong standard method," applied with adapted bases, solves certain nonabelian HSPs in quantum polynomial time that cannot be solved using measurements in random bases or "forgetful" abelian approaches.

While we are still very far from an algorithm for HSP in the symmetric group $S_{n}$ or for Graph Automorphism, a global understanding of the power of strong Fourier sampling remains an important goal. Perhaps the next class of groups to try beyond the affine and $q$-hedral groups are matrix groups such as $\operatorname{PSL}_{2}(p)$, whose maximal 
subgroups are isomorphic to $A_{p}$, and which include one of the infinite families of finite simple groups.

Appendix A. Notes on exponential sums. The basic Gauss sum bounds the inner products of additive and multiplicative characters of $\mathbb{F}_{p}$, the finite field of prime cardinality $p$. Definitive treatments appear in [19, section 5] and [17]. Considering $\mathbb{F}_{p}$ as an additive group with $p$ elements, we have $p$ additive characters $\chi_{s}: \mathbb{F}_{p} \rightarrow \mathbb{C}$, for $s \in \mathbb{F}_{p}$, given by $\chi_{s}: z \mapsto \omega_{p}^{s z}$, where, as above, $\omega_{p}=\mathrm{e}^{2 \pi i / p}$ is a primitive $p$ th root of unity. Likewise considering the elements of $\mathbb{F}_{p}^{*}=\mathbb{F}_{p} \backslash\{0\}$ as a multiplicative group, we have $p-1$ characters $\psi_{t}: \mathbb{F}_{p}^{*} \rightarrow \mathbb{C}$, for $t \in \mathbb{F}_{p}^{*}$, given by $\psi_{t}: g^{z} \mapsto \omega_{p-1}^{t z}$, where $\omega_{p-1}=\mathrm{e}^{2 \pi i /(p-1)}$ is a primitive $(p-1)$ th root of unity and $g$ is a multiplicative generator for the (cyclic) group $\mathbb{F}_{p}^{*}$.

With this notation the basic Gauss sum is the following.

THEOREM 8. Let $\chi_{s}$ be an additive character and $\psi_{t}$ a multiplicative character of $\mathbb{F}_{p}$. If $s \neq 0$ and $t \neq 1$, then

$$
\left|\sum_{z \in \mathbb{F}_{p}^{*}} \chi_{s}(z) \psi_{t}(z)\right|=\sqrt{p}
$$

Otherwise

$$
\sum_{z \in \mathbb{F}_{p}^{*}} \chi_{s}(z) \psi_{t}(z)= \begin{cases}p-1 & \text { if } s=0, t=1 \\ -1 & \text { if } s=0, t \neq 1 \\ 0 & \text { if } s \neq 0, t=1\end{cases}
$$

See [19, section 5.11] for a proof.

This basic result has been spectacularly generalized. In the body of the paper we require bounds on additive characters taken over multiplicative subgroups of $\mathbb{F}_{p}^{*}$. Such sums are discussed in detail in [17]. The specific bound we require is the following.

TheOREM 9. Let $\chi_{t}$ be a nontrivial additive character of $\mathbb{F}_{p}$ and $a \in \mathbb{F}_{p}^{*}$ an element of multiplicative order $q$. Then

$$
\sum_{z=0}^{q-1} \chi_{t}\left(a^{z}\right)= \begin{cases}O\left(p^{1 / 2}\right) & \text { if } q \geq p^{2 / 3} \\ O\left(p^{1 / 4} q^{3 / 8}\right) & \text { if } p^{1 / 2} \leq q \leq p^{2 / 3} \\ O\left(p^{1 / 8} q^{5 / 8}\right) & \text { if } p^{1 / 3} \leq q \leq p^{1 / 2}\end{cases}
$$

See [17, section 2] for a proof.

Note that in the body of the paper, we use $\mathbb{Z}_{p}$ to denote the additive group of integers modulo $p$ and $\mathbb{Z}_{p}^{*}$ to denote the multiplicative group of integers modulo $p$.

Acknowledgments. We are grateful to Wim van Dam, Julia Kempe, Greg Kuperberg, Frederic Magniez, Martin Rötteler, and Miklos Santha for helpful conversations and to Sally Milius and Tracy Conrad for their support.

\section{REFERENCES}

[1] D. Bacon, A. Childs, And W. VAn Dam, From optimal measurement to efficient quantum algorithms for the hidden subgroup problem over semidirect product groups, in Proceedings of the 46th Annual IEEE Symposium on Foundations of Computer Science, 2005, pp. 469478.

[2] R. BEALS, Quantum computation of Fourier transforms over symmetric groups, in Proceedings of the 29th Annual ACM Symposium on the Theory of Computing, 1997, pp. 48-53. 
[3] E. Bernstein And U. VAzirani, Quantum complexity theory (preliminary abstract), in Proceedings of the 25th Annual ACM Symposium on the Theory of Computing, 1993, pp. 1120.

[4] W. van Dam, S. HALlgren, AND L. Ip, Quantum algorithms for some hidden shift problems, in Proceedings of the Fourteenth Annual ACM-SIAM Symposium on Discrete Algorithms, 2003, pp. 489-498.

[5] I. B. DAMGÅRD, On the randomness of Legendre and Jacobi sequences, in Advances in Cryptology - CRYPTO '88, Lecture Notes in Comput. Sci. 403, Springer-Verlag, Berlin, 1990, pp. 163-172.

[6] M. ETTINGER AND P. HøYeR, On quantum algorithms for noncommutative hidden subgroups, Adv. in Appl. Math., 25 (2000), pp. 239-251.

[7] M. EtTinger, P. HøYer, And E. Knill, The quantum query complexity of the hidden subgroup problem is polynomial, Inform. Process. Lett. 91 (2004), pp. 43-48.

[8] K. Friedl, G. Ivanyos, F. Magniez, M. Santha, and P. Sen, Hidden translation and orbit coset in quantum computing, in Proceedings of the 35th Annual ACM Symposium on Theory of Computing, 2003, pp. 1-9.

[9] W. Fulton and J. Harris, Representation Theory: A First Course, Grad. Texts in Math. 129, Springer-Verlag, London, 1991.

[10] M. Grigni, L. J. Schulman, M. Vazirani, and U. Vazirani, Quantum mechanical algorithms for the nonabelian hidden subgroup problem, in Proceedings of the 33rd Annual ACM Symposium on Theory of Computing, 2001, pp. 68-74.

[11] L. Hales and S. Hallgren, Quantum Fourier sampling simplified, in Proceedings of the 31st Annual ACM Symposium on Theory of Computing, 1999, pp. 330-338.

[12] L. Hales and S. Hallgren, An improved quantum Fourier transform algorithm and applications, in Proceedings of the 41st Annual IEEE Symposium on Foundations of Computer Science, 2000, pp. 515-525.

[13] S. Hallgren, A. Russell, And A. TA-Shma, Normal subgroup reconstruction and quantum computation using group representations, in Proceedings of the 32nd Annual ACM Symposium on Theory of Computing, 2000, pp. 627-635.

[14] P. HøYER, Efficient Quantum Transforms, http://arxiv.org/abs/quant-ph/9702028 (1997).

[15] G. Ivanyos, F. Magniez, And M. Santha, Efficient quantum algorithms for some instances of the non-abelian hidden subgroup problem, Internat. J. Found. Comput. Sci., 14 (2003), pp. $723-740$.

[16] R. JozSA, Quantum Factoring, Discrete Logarithms and the Hidden Subgroup Problem, http://arxiv.org/quant-ph/0012084 (2000).

[17] S. V. Konyagin And I. E. ShParlinski, Character Sums with Exponential Functions and Their Applications, Cambridge Tracts in Math. 136, Cambridge University Press, Cambridge, UK, 1999.

[18] G. KuPERBERG, A subexponential-time quantum algorithm for the dihedral hidden subgroup problem, SIAM J. Comput., 35 (2005), pp. 170-188.

[19] R. Lidl And H. Niederreiter, Finite Fields, Encyclopedia Math. Appl. 20, Cambridge University Press, Cambridge, UK, 1997.

[20] C. Moore, D. Rockmore, and A. Russell, Generic quantum Fourier transforms, in Proceedings of the Fifteenth Annual ACM-SIAM Symposium on Discrete Algorithms, 2004, pp. $771-780$.

[21] C. Moore And A. Russell, For distinguishing conjugate hidden subgroups, the pretty good measurement is as good as it gets, Quantum Inf. Comput., to appear.

[22] M. Roetteler and T. Beth, Polynomial-Time Solution to the Hidden Subgroup Problem for a Class of Non-Abelian Groups, http://arxiv.org/abs/quant-ph/9812070 (1998).

[23] J. Rotman, An Introduction to the Theory of Groups, Grad. Texts in Math. 148, SpringerVerlag, London, 1994.

[24] J.-P. SerRe, Linear Representations of Finite Groups, Grad. Texts in Math. 42, SpringerVerlag, London, 1977.

[25] P. W. SHOR, Polynomial-time algorithms for prime factorization and discrete logarithms on a quantum computer, SIAM J. Comput., 26 (1997), pp. 1484-1509.

[26] D. R. Simon, On the power of quantum computation, SIAM J. Comput., 26 (1997), pp. 14741483. 